\title{
Impact of manufacturing errors of ball screw system on performance characteristics
}

\author{
Alexander Denisenko ${ }^{1, *}$ \\ ${ }^{1}$ Samara State Technical University, Samara, Russian Federation
}

\begin{abstract}
To convert rotary motion into translational motion in modern metal-cutting machine tools, screw-nut rolling transmissions are widely used. Special requirements are placed on the stability of the operational characteristics of the screw-nut rolling transmission. The influence of the accuracy of manufacturing screw-nut transmission on the main transmission characteristics: axial stiffness and the moment of idling is considered. When analyzing the quality of the manufacture of the transmission, the following manufacturing errors were taken into account: the pitch errors of the screw and nut threads; errors in thread diameter of screw and nut; deviation of the thread profile of the screw and nut; the error of the diameter of the ball. Since these errors are independent random variables, on the basis of the basic propositions of the theory of probability, analytical dependencies were obtained to determine the coefficients connecting the variances of the design parameters and the variance of the probabilistic distribution of the axial force and the idling torque. Based on the calculation results from the obtained dependencies, it was concluded that the errors in the thread pitch of the screw and nut have a prevailing influence on the dispersion of the probabilistic distribution of the axial force (and, consequently, the axial stiffness) and the idling torque. The results obtained allow us to outline further ways of improving the transmission of the screw-nut rolling.
\end{abstract}

\begin{abstract}
A feature of the design of the drives for the supply of a significant number of CNC machines is the presence of feedback with an incompletely closed loop, when feedback only covers the torsion part of the mechanical chain of the drive. In connection with this, the role of the mechanism of the transformation of the rotary motion into the translational mechanism is very important in shaping the characteristics of the drives. In modern metal-cutting machines, the transmission of screw-nut rolling is widely used as such a mechanism.

The most important performance characteristics of the transmission of screw-nut rolling are the axial stiffness and the moment of idling. They affect the provision of the necessary dynamic characteristics of the feed drives, without which it is impossible to create modern precision CNC machines [1-9].

In connection with these design issues, methods of calculation, development of ways to improve the transmission of screw-nut rolling, a lot of attention has recently been paid [1, 10-12].
\end{abstract}

\footnotetext{
* Corresponding author: ask@samgtu.ru
} 
To determine the axial stiffness of the transmission representing the ratio of the force acting on the screw (nut) $P$ to the force $\delta$, you can use addiction [13]

$$
P=\sum_{i=1}^{z}\left(\frac{\delta_{i}}{2 C_{i}}\right)^{3 / 2} \sin ^{5 / 2} \alpha_{i} \cos ^{5 / 2} \lambda_{i}
$$

where $\delta_{i}$ - the relative axial displacement of the screw and nut for the $i$ - th ball; $C_{i}$ coefficient, depending on the parameters of the contacting bodies; $z$ - the number of working balls in the nut.

Angle of contact i-th ball $\alpha_{i}$ in the transmission collected with preload can be defined as the sum of two terms [15]: the first term is determined by the geometric parameters of the transmission (the initial contact angle with the gap equal to zero), the second term takes into account the change in angle $\alpha_{i}$ due to deformation of the elements transmission under the action of prestressing forces:

$$
\alpha=\arccos W_{i}+\arccos \left(N_{i} / \sqrt{B_{i}}\right)
$$

where

$$
\begin{gathered}
F_{i}=r_{s i}+r_{n i}-d_{b i} ; W_{i}=\frac{r_{s i}+r_{n i}-0,5\left(d_{n i}-d_{s i}\right)}{F_{i}} ; N_{i}=0,5\left(\delta_{i} \sin \alpha_{0 i} \cos \lambda+F_{i}\right) ; \\
B_{i}=0,25 \delta_{i}^{2} \cos ^{2} \lambda+0,5 \delta_{i} F_{i} \sin \alpha_{0 i} \cos \lambda+0,25 F_{i}^{2}
\end{gathered}
$$

$r_{s}$ and $r_{n}$ - the radii of the thread groove of the screw and nut; $d_{s}$ - internal thread diameter of the screw; $d_{n}$ - internal thread diameter of the nut; $d_{b}$ - diameter of the ball.

The moment of idling is determined mainly by the rolling friction of the balls in the working area. The moment of friction of a single ball in a transmission operating in the rotaryto- translational motion mode can be found by formula [13]

$$
M_{i}=0,5 P_{i} d_{c} \sin \alpha_{i} \sin (\lambda \pm \rho),
$$

where $d_{c}$ - the diameter of the contact of the balls with the screw; $\rho$-reduced friction angle.

Since the sign in the trigonometric function $\sin (\lambda \pm \rho)$ in the above formula depends on the direction of rotation of the screw, then for the nut assembled from the two half-nuts, for one half-nut the sign $(+)$ is taken, and for the other $(-)$.

Using the dependence connecting $d_{c i}$ and the basic transmission parameters [13], we can obtain an expression for determining the moment of the cold running of the screw-nut rolling transmission in the form:

$$
M=R \sum_{i=1}^{z}\left(\frac{\delta_{i}}{2 C_{i}}\right)^{3 / 2}\left[\frac{d_{n i}+d_{s i}}{2}+\left(r_{s i}-r_{n i}\right)\left(1-\cos \alpha_{0 i}\right)-d_{b} \cos \alpha_{i}\right] \sin ^{5 / 2} \lambda_{i},
$$

where $R=0,5 \cos ^{3 / 2} \lambda \sin (\lambda \pm \rho)$.

As can be seen from the dependencies for determining the axial force $P$ and the idle speed $M$, these characteristics depend on many transmission parameters. The actual values of these parameters always have some deviations from the nominal value within the given manufacturing tolerances, which are of a random nature. In this case it is quite legitimate to 
talk about the mathematical expectation and variance of the probability distribution of each parameter of the transmission elements. And since the constituent transmission elements (screw, nut, balls) have parameters that obey the laws of probability theory, then transmission performance characteristics, such as stiffness and the moment of idling, also have a random character and have their own mathematical expectation and their variance.

The actual values of the parameters of the screw-nut transmission elements are determined by the primary errors, which include the following manufacturing errors: the error of the thread pitch of screw $\Delta t_{s}$ and nut $\Delta t_{n}$; errors in thread diameter of screw $\Delta d_{s}$ and nut $\Delta d_{n}$; deviation of thread profile of screw $\Delta r_{s}$ and nut $\Delta r_{n}$; the error of the diameter of the ball $\Delta d_{b}$.

The primary transmission errors are independent random variables. In this case, one can use one of the theorems [15], according to which the mathematical expectation of a random variable $z=f\left(z_{1}, z_{2}, \ldots, z_{k}\right)$ for independent random variables $z_{i} \quad(i=1,2, \ldots, k)$ can be determined from the dependence

$$
E(z) \approx f\left[E\left(z_{1}\right), E\left(z_{2}\right), \ldots, E\left(z_{k}\right)\right],
$$

and the variance on the dependence

$$
D(z) \approx \sum_{i=1}^{k}\left(\frac{\partial f}{\partial z_{i}}\right)^{2} D\left(z_{i}\right)
$$

where $E\left(z_{i}\right)$ and $D\left(z_{i}\right)$ are the mathematical expectation and variance of the random variable $z_{i}$. The derivatives are taken at the point $\left[E\left(z_{1}\right), E\left(z_{2}\right), \ldots, E\left(z_{k}\right)\right]$.

Derivatives $\frac{\partial f}{\partial z_{i}}$ show the degree of influence of the error of the i-th parameter on the error of the output characteristic. The larger the absolute value of $\left|\frac{\partial f}{\partial z_{i}}\right|$, the stronger this effect, the greater the accuracy of the i-th parameter.

Based on the analysis of the above dependencies, the dispersion of the probabilistic distribution of the axial force acting on the transmission of the screw-nut rolling can be determined as follows:

$$
D(P)=K_{1} D\left(t_{s}\right)+K_{2} D\left(t_{n}\right)+K_{3} D\left(d_{b}\right)+K_{4} D\left(d_{s}\right)+K_{5} D\left(d_{n}\right)+K_{6} D\left(r_{s}\right)+K_{7} D\left(r_{n}\right) .
$$

The coefficients $K_{i}(i=1,2, \ldots, 7)$ show the degree of influence of the corresponding parameters on the variance of the probability distribution of the axial force and are calculated according to the rules given above:

$$
\begin{aligned}
& K_{1}=K_{2}=\frac{\mu}{4}\left(\frac{2 C}{\delta_{p}}\right)^{2}\left[\frac{3 \sin \alpha}{C}-5 G\left(\frac{\delta_{p}}{2 C}\right) \cos \alpha \cos \lambda\right]^{2} ; \\
& K_{3}=\mu\left\{\frac{2 T}{d_{b}^{2}}+5 \cos \alpha\left[\frac{2 r-0,5\left(d_{n}-d_{s}\right)}{\left(2 r-d_{b}\right)^{2} \sqrt{1-Q^{2}}}+S\right]\right\}^{2} ;
\end{aligned}
$$




$$
\begin{aligned}
& K_{4}=K_{5}=\frac{25 \mu \cos ^{2} \alpha}{4\left(2 r-d_{b}\right)^{2}\left(1-Q^{2}\right)} ; \\
& K_{6}=K_{7}=\mu\left\{\frac{T}{2 r^{2}}+5 \cos \alpha\left[\frac{0,5\left(d_{n}-d_{s}\right)-d_{b}}{\left(2 r-d_{b}\right)^{2} \sqrt{1-Q^{2}}}+S\right]\right\}^{2},
\end{aligned}
$$

where

$$
\begin{aligned}
& \mu=\frac{z^{2}}{4}\left(\frac{\delta_{p}}{2 C}\right)^{3} \sin ^{3} \alpha \cos ^{5} \lambda ; T=\frac{m_{\delta} \sin \alpha}{C E^{2 / 3}}\left(\frac{2}{d_{b}}-\frac{1}{r}\right)^{-2 / 3} ; C=\frac{m_{\delta}}{E^{2 / 3}}\left(\frac{2}{d_{b}}-\frac{1}{r}\right)^{1 / 3} ; \\
& \left.S=\frac{B^{1 / 2}-0,5 N B^{-1 / 2}\left(\delta_{p} \cos \lambda \sin \alpha_{0}+2 r-d_{b}\right)}{2 B \sqrt{1-N B^{-1 / 2}}} ; N=0,5 \mid \delta_{p} \sin \alpha_{0} \cos \lambda+\left(2 r-d_{b}\right)\right] ; \\
& G=\frac{B^{1 / 2} \sin \alpha_{0}-0,5 N B^{-1 / 2}\left[\delta_{p} \cos \lambda+\sin \alpha_{0}\left(2 r-d_{b}\right)\right]}{B \sqrt{1-N B^{-1 / 2}}} ; Q=\frac{2 r-0,5\left(d_{n}-d_{s}\right)}{2 r-d_{b}} ; \\
& B=0,25 \delta_{p}^{2} \cos ^{2} \lambda+0,5 \delta_{p}\left(2 r-d_{b}\right) \cos \lambda \sin \alpha_{0}+0,25\left(2 r-d_{b}\right)^{2} ;
\end{aligned}
$$

$z$ - number of balls in the nut; $m_{\delta}$ - coefficient, which is a function of the ratio of the principal curvatures of the ball and the profile of the nut (screw) [13]; $r$ - nominal value of radii $r_{s}$ and $r_{n} ; \delta_{p}$ - the preload in the transmission of the screw-nut rolling; $E$ - the modulus of elasticity of the material of the contacting bodies.

Similarly, for the dispersion of the probability distribution of the no-load moment:

$$
D(M)=K_{1}^{M} D\left(t_{s}\right)+K_{2}^{M} D\left(t_{n}\right)+K_{3}^{M} D\left(d_{b}\right)+K_{4}^{M} D\left(d_{s}\right)+K_{5}^{M} D\left(d_{n}\right)+K_{6}^{M} D\left(r_{s}\right)+K_{7}^{M} D\left(r_{n}\right) .
$$

The coefficients $K_{i}^{M}(i=1,2, \ldots, 7)$ are determined from the following dependences

$$
\begin{aligned}
& K_{1}^{M}=K_{2}^{M}=\theta\left(\frac{2 C}{\delta_{p}}\right)^{2}\left[\frac{3 \tau \sin \alpha}{4 C}-0,5 G v\left(\frac{\delta_{p}}{2 C}\right) \cos \lambda\right]^{2} ; \\
& K_{3}^{M}=\theta\left\{\frac{T \tau}{d_{b}^{2}}+v\left[\frac{2 r-0,5\left(d_{n}-d_{s}\right)}{\left(2 r-d_{b}\right)^{2} \sqrt{1-Q^{2}}}-S\right]-\sin \alpha \cos \alpha\right]^{2} ; \\
& K_{4}^{M}=K_{5}^{M}=\theta\left[\frac{v}{2\left(2 r-d_{b}\right)^{2} \sqrt{1-Q^{2}}}+0,5 \xi \sin \alpha\right]^{2} ;
\end{aligned}
$$




$$
K_{6}^{M}=K_{7}^{M}=\theta\left\{\xi \sin \alpha\left(1-\cos \alpha_{0}\right)-\frac{T \tau}{4 r^{2}}+v\left[\frac{0,5\left(d_{n}-d_{s}\right)-d_{b}}{\left(2 r-d_{b}\right)^{2} \sqrt{1-Q^{2}}}+S\right]\right\}^{2}
$$

where

$$
\begin{aligned}
& \theta=\left(\frac{\delta_{p}}{2 C}\right) R^{2} z^{2} \sin ^{3} \alpha ; \tau=d_{0}-d_{b} \cos \alpha ; v=2.5 \cos \alpha_{0}\left(d_{0}-d_{b} \cos \alpha\right)+d_{b} \sin ^{2} \alpha ; \\
& \xi=\left\{\begin{array}{lllll}
+1 & \text { for } & K_{5}^{M} & \text { and } & K_{6}^{M} \\
-1 & \text { for } & K_{4}^{M} & \text { and } & K_{7}^{M}
\end{array} ;\right.
\end{aligned}
$$

$d_{0}$ - the nominal diameter of the screw-nut transmission.

The coefficients $K_{i}$ and $K_{i}^{M}$, calculated from the dependences obtained, allow us to estimate the degree of influence of the i-th parameter on the variance of the probability distribution of the operational characteristics of the screw-nut rolling transmission and to outline the most effective ways of their improvement.

As an example, the table shows the results of calculating the coefficients $K_{i}$ and $K_{i}^{M}$ for the transmission of a screw-nut rolling 50x10 for different values of the preload.

Table 1. The coefficients $K_{i}$ and $K_{i}^{M}$ for the transmission of a screw-nut rolling 50x10

\begin{tabular}{|r|c|r|r|r|r|r|r|}
\hline $\begin{array}{r}\text { Preload } \\
\delta_{p}, \mathrm{~mm}\end{array}$ & Coefficient & $\begin{array}{r}i=1 \\
i=2\end{array}$ & $i=3$ & $i=4$ & $i=5$ & $i=6$ & $i=7$ \\
\hline \multirow{2}{*}{0,004} & $K_{i} \cdot 10^{-7}$ & 396.587 & 0.439 & 0.145 & 0.145 & 0.102 & 0.102 \\
\cline { 2 - 8 } & $\left(K_{i}^{M} / R^{2}\right) \cdot 10^{-10}$ & 838.830 & 0.436 & 0.328 & 0.330 & 0.226 & 0.227 \\
\hline \multirow{2}{*}{0,006} & $K_{i} \cdot 10^{-7}$ & 622.668 & 1.523 & 0.492 & 0.492 & 0.355 & 0.355 \\
\cline { 2 - 8 } & $\left(K_{i}^{M} / R^{2}\right) \cdot 10^{-10}$ & 1319.237 & 1.457 & 1.116 & 1.123 & 0.787 & 0.790 \\
\hline \multirow{2}{*}{0,008} & $K_{i} \cdot 10^{-7}$ & 870.243 & 3.663 & 1.175 & 1.175 & 0.874 & 0.874 \\
\cline { 2 - 8 } & $\left(K_{i}^{M} / R^{2}\right) \cdot 10^{-10}$ & 1847.065 & 3.406 & 2.672 & 2.688 & 1.944 & 1.952 \\
\hline
\end{tabular}

Taking into account the fact that the variances of the probability distribution of all the parameters of the transfer of the screw-nut rolling $D\left(t_{s}\right), D\left(t_{n}\right), D\left(d_{b}\right), D\left(d_{s}\right), D\left(d_{n}\right)$, $D\left(r_{s}\right), D\left(r_{n}\right)$ according to the technical conditions for its production are quantities of the same order, it follows from the calculation data that on the dispersion of the probabilistic distribution of the axial force (and, consequently, stiffness) and the no-load moment the prevailing influence is made by the errors in the thread pitch of the screw and nut, and, as can be seen from the table, this ratio practically does not change with the change in the preload. This conclusion is confirmed by the results given in $[16,17]$. 


\section{Conclusions}

1. The obtained dependences allow estimating the degree of influence of the parameters of the screw-nut rolling element on its performance characteristics.

2. One of the reserves to improve the accuracy and dynamic characteristics of the CNC machine feeders is the reduction in pitch errors of the thread of the screw and the nut of the screw-nut transmission.

\section{References}

1. V.V. Kozyrev, M.V. Kozhevnikova, System for analysis and synthesis of the power part of mechatronic modules, Mechatronics, automation, control, v. 2, pp. 28-32 (2009)

2. L. Fuhua, J. Yao, L. Tiemin, D. Yunsong, An improved dynamic model of preloaded ball screw drives considering torque transmission and its application to frequency analysis, Advances in Mechanical Engineering, v. 9(7), pp. 1-11 (2017)

3. J. Liscouët, M. Budinger, S. Orieux, J.-C. Maré, Modelling approach for the SimulationBased Preliminary Design of Power Transmissions, Mechanism and Machine Theory, v. 46 (3), pp. 276-289 (2011)

4. A.V. Kuznetsov, Modeling of multicomponent dynamic systems using the example of a ball screw drive, Engineering - from theory to practice, v. 14, pp. 25-29 (2012)

5. L.W. Novotny, J. Marek, T. Marek, Issue of Rigidity of the Ball Screw Nut Exposed to Bending Stress during Bending Stress of the Ball Screw, Applied Mechanics and Materials, v. 821, pp. 372-377, (2016)

6. R. Neugebauer, S. Ihlenfeldt, A.Hellmich, H.Schlegel, Modelling feed drives based on natural excitation - improving accuracy, CIRP Annals. Manufacturing Technology, v. 66, no. 1, pp. 369-372, (2017)

7. K. Erkorkmaz, A. Kamalzadeh, High Bandwidth Control of Ball Screw Drives, CIRP Annals. Manufacturing Technology, v. 55, issue 1, pp.393-398, (2006)

8. G. Pritschow, N. Croon, Ball screw drives with enhanced bandwidth by modification of the axial bearing, CIRP Annals. Manufacturing Technology, v. 62, issue 1, pp.383-386, (2013)

9. J. Zhang, H. Zhang, C. Du, W. Zhao, Research on the dynamics of ball screw feed system with high acceleration, International Journal of Machine Tools and Manufacture, v. 111, pp.9-16, (2016)

10. B.M. Isnairov, S.N. Aksenov, S.A. Shilenko, Methods for increasing the load capacity of ball screw transmission (review), Materials of the 3rd International Scientific and Practical Conference "Modern Materials, Technology and Technology", pp. 163-164, (2013)

11. V.V. Bushuyev, V.V. Molodtsov, Influence on the dynamic characteristics of drives for feeding the elastic properties of their mechanisms, STIN, v. 5. pp. 8-14 (2016)

12. B.M. Iznayrov, A.N. Vasin, Technological Methods for Improving the Performance Properties of Screw Gears (Survey of Research), Technology of Mechanical Engineering, v. 9. pp. 26-31 (2016)

13. D.N. Reshetov, Details and mechanisms of metal-cutting machine (Moscow, Mechanical Engineering, 1972)

14. P.P. Shardyko. Investigation of the rigidity and uniformity of the movement of ball screw mechanisms, $\mathrm{PhD}$ dissertation, Moscow, 1975 
15. H.B. Kordonskii, Applications of the theory of probability in engineering (MoscowLeningrad, Fizmatgiz, 1963)

16. A.N. Vasin, B.M. Ignatyrov, G.Kh. Mukatova, A.K. Bondarev, Investigation of the influence of the technological step error on the rigidity of a ball screw transmission, Materials of the X International Scientific and Practical Conference "Fundamental and Applied Sciences Today", pp. 109-112 (2016)

17. B.M. Ignatyev, G.Kh. Mukatova, A.K. Bondarev, Experimental Investigations of the Impact of Technological Step Errors on the Axial Deformation of a Ball Screw Transmission, Materials of the International Scientific and Practical Conference "Role of Innovations in Transformation of Contemporary Science", pp. 32-35 (2016). 